
16(13) : 3203-3208 (2020), copyright ( 2020 The Royal Society of Chemistry. To access the final edited and published work see https://doi.org/10.1039/C9SM02471A

\title{
Effect of environmental humidity on the ionic transport of poly(ethylene oxide) thin films by local dielectric spectroscopy
}

\author{
Paul Markus ${ }^{1}$, Daniel E. Martínez-Tong ${ }^{2,3, *}$, Georg Papastavrou ${ }^{1,4}$, Angel Alegria ${ }^{2,3, *}$ \\ ${ }^{1}$ Physical Chemistry II. Faculty of Biology. University of Bayreuth. Universitätsstraße 30, 95440 \\ Bayreuth - Germany. \\ ${ }^{2}$ Departamento de Física de Materiales, Basque Country University (UPV/EHU). P. Manuel de Lardizábal \\ 3, E-20018 San Sebastián - Spain. \\ ${ }^{3}$ Centro de Física de Materiales (CSIC - UPV/EHU). P. Manuel Lardizábal 5, E-20018 San Sebastián - \\ Spain. \\ ${ }^{4}$ Bavarian Battery Research Center at the University of Bayreuth (BayBatt). University of Bayreuth - \\ Germany. \\ Corresponding authors: danielenrique.martinezt@ehu.eus
}

angel.alegria@ehu.eus

\begin{abstract}
The effect of humidity on the ionic transport in the amorphous phase of poly(ethylene oxide) thin films has been studied by via local dielectric spectroscopy. We explored a controlled humidity range between $15 \% \mathrm{RH}$ and $50 \% \mathrm{RH}$. AFM-based local dielectric imaging allowed to obtain simultaneously the thin films topography and the corresponding dielectric contrast maps. No humidity effect on the film topography was observed whereas large variation of the dielectric signal could be detected. In addition, we observed a clear dielectric contrast in different locations on the thin film surface. At selected regions with high contrast in the dielectric maps, we performed nanoDielectric Spectroscopy (nDS) measurements covering the frequency range from $5 \mathrm{~Hz}$ to $100 \mathrm{kHz}$. By modeling these spectroscopy results, we quantified the conductivity of the amorphous phase of the semicrystalline poly(ethylene oxide) films. The crystalline fraction of the PEO thin films was extracted and found to be about $36 \%$, independently of humidity. However, the average conductivity increased by a factor of 25 from $2 \times 10^{-}$ 10 to $5 \times 10^{-9} \mathrm{~S} / \mathrm{cm}$, by changing environmental humidity in the explored $\% \mathrm{RH}$ range.
\end{abstract}




\section{Introduction}

In times where electromobility and sustainable chemistry are of central interest, energy storage devices based on solid-state electrolytes are the key for developing future technologies. In particular, polymers ${ }^{1-3}$ and nanocomposites ${ }^{4-6}$ feature favorable ion conduction properties for their application as electrolyte membranes. These materials have good mechanical properties, thermal tolerance, and electrochemical stability, compared to their liquid- and gel-based counterparts. Currently, the most promising materials for the development of polymer-batteries are the poly(ethylene oxide) (PEO) and its family of derivatives. As summarized by Jiang et al, ${ }^{7}$ PEO shows good chain flexibility, superior electrochemical stability to lithium metal, low glass transition temperature, and excellent solubility with conductive lithium salts. However, at room temperature, PEO is a semicrystalline material where amorphous and crystalline phases coexist at the nanoscale. The PEO crystals provide the material with good mechanical properties at room temperature; however, they also act as hindrances for ion conduction..$^{7-9}$ In other words, a blocking of charges takes place at the internal phase boundaries of PEO, leading to low film conductivities, which usually increase by several orders of magnitude after doping with ion salts. ${ }^{10}$

The build-up of charges at the interfaces is manifested via a dielectric relaxation in the multiphase material, known as Maxwell-Wager-Sillars (MWS) relaxation. ${ }^{11,12}$ In dielectric experiments on PEO, the MWS dielectric relaxation signal appears at room temperature, in a frequency window of $5-10^{5} \mathrm{~Hz} \cdot{ }^{9,13}$ This frequency range is fully covered using nanoDielectric Spectroscopy (nDS). This local technique combines the power of broadband dielectric spectroscopy (BDS) with the lateral resolution of scanning probe microscopes (SPM). ${ }^{14-16}$ Lately, a variety of SPM techniques based on electrical mode operation have emerged and evolved. ${ }^{17}$ These methods facilitate the 
local measurement of sample properties, such as conductivity, permittivity, and surface potential, with high lateral resolution. Particularly, in $\mathrm{nDS}$, the dielectric response of a sample can be measured by means of analyzing the electrostatic interaction force with an atomic force microscopy (AFM) probe. ${ }^{18}$ Thereby, dielectric relaxation processes can be studied in a thin film material with nanoscale resolution.

In a previous study, we explored the dielectric properties of PEO thin films obtained using different solvents, under dry atmospheric conditions, via nDS. ${ }^{9}$ These AFM-based measurements allowed to extract important physical parameters of the films, such as the volume fraction of the ion-blocking crystalline phase and the conductivity of the PEO amorphous phase at room temperature. Addressing the changes in film structure and properties in dependence of humidity conditions is of outmost importance when considering any possible application of PEO thin films as ion transporting material. ${ }^{19}$ Therefore, in the present work we focus on the influence of the humidity on the ionic transport of PEO thin films by using a similar experimental approach but with an improved methodology providing higher sensitivity. The here-presented approach allowed us detecting the local dielectric relaxation of the PEO thin films, depending on the probe position. Moreover, we confirmed the strong impact of humidity on the measured signal, whose characteristic frequency changes by a factor of 25 over the explored humidity range. We attributed this change to the variation of the ionic conductivity of the amorphous phase, which forms a major part of the PEO films.

\section{Experimental section}

Materials. Poly(ethylene oxide) (PEO) has been purchased from Sigma-Aldrich and was used as received. The material had a molecular weight $M_{\mathrm{w}}=25 \mathrm{~kg} / \mathrm{mol}$, as determined by viscosity measurements. Polymer thin films were prepared by spin coating. PEO solutions were spin casted onto silicon wafers (3000 rpm, 2 min), using 
tetrahydrofuran as solvent (20 $\mathrm{mg} / \mathrm{mL}$ concentration). Prior to spin coating, the solutions were filtered using PTFE syringe filters $(0.2 \mu \mathrm{m})$. All thin films were subjected to a vacuum treatment $\left(10^{-6}\right.$ torr) to remove possible residual solvent. Prior imaging, all samples were annealed at $100{ }^{\circ} \mathrm{C}$ for $15 \mathrm{~min}$, under a continuous $\mathrm{N}_{2}$ flow. This procedure allowed erasing any thermal history, and suppressed solvent influence on morphology and other properties, as previously reported. ${ }^{9,20}$

Methods. The surface topography of the thin polymer films has been acquired by Atomic Force Microscopy (AFM) measurements. All measurements were performed using a Multimode AFM equipped with a Nanoscope V controller and the Signal Access Module III (Bruker). We used the $\mathrm{Cr} / \mathrm{Pt}$ coated conducting probes Tap300E-G from BudgetSensors (typical resonance frequency $\sim 250 \mathrm{kHz}$, and spring constant $k \sim 25$ $\mathrm{N} / \mathrm{m}$ ). We used a J-scanner that allowed controlling the sample stage temperature. Moreover, we probed the material's local dielectric response by analyzing the cantilever oscillations induced by the interaction with the sample, due to an $\mathrm{AC}$ electric field. For these electrical measurements, we used an external lock-in amplifier SR-865A equipped with a SIM983 scaling amplifier (Stanford Research Systems). This approach allows imaging maps of the dielectric response of the sample, similar to Electric Force Microscopy (EFM), with the extra capabilities of performing spectroscopy measurements at specific locations on the surface, by varying the frequency $(f)$ of the AC voltage applied to the tip. ${ }^{9,21,22}$ In both cases, we analyzed the second harmonic component of the electrical force, i.e., that at a frequency double than that of the applied voltage. This force component is related with the complex capacitance of the probesample system $C^{*}$, which depends on the polymer film's dielectric function: $\varepsilon^{*}(\omega)=\varepsilon^{\prime}(\omega)$ $-i \varepsilon^{\prime \prime}(\omega)$, as: $^{21}$ 


$$
F_{2 \omega}^{*}(t)=-\frac{1}{4} \cdot \frac{\partial C^{*}}{\partial z} V_{0}^{2} \cos (2 \omega t)
$$

In eq (1), $z$ is the coordinate along which the tip-sample distance is measured, $V_{0}$ is the amplitude of the AC voltage and $\omega=2 \pi f$, with the electric field frequency $f$. The photodiode signal, encoding the probe motion due to the probe-sample interaction, was analyzed using the lock-in amplifier to obtain the phase $(\theta)$ of the probe oscillations at $2 \omega$.

In the here-reported experiments, all the AFM measurements were performed under a humidity-controlled atmosphere. The implementation of the used set-up consisted of two gas flow controllers connected to a compressed nitrogen line in a parallel fashion. One stream contained dry $\mathrm{N}_{2}$ gas, while the other one was guided through a bubbler bottle filled with pure water. By adjusting the gas flow ratio between the dry and wet streams, an environment with controlled humidity could be obtained. The combined gas stream was guided to the AFM measurement chamber, where a humidity sensor (SHT31, Sensirion AG) recorded the actual value of relative humidity. A homemade Igor Pro (WaveMetrics, Inc.) procedure was used to implement a PID (proportionalintegral-derivative) algorithm for adjusting the gas flow ratio. Our setup allowed for controlling the humidity in a range between $15-65 \% R H$, with an estimated accuracy better than $0.1 \% R H$. By the real-time evaluation of the humidity sensor data, we found that upon changing the \% RH set-point value, a period of 15 min was usually sufficient for environment equilibration, which provided good long-term stability.

The thickness of the PEO thin films was also determined via AFM, by measuring the step between the polymer film and the supporting substrate. These measurements were performed at ambient conditions $\left(25^{\circ} \mathrm{C}, 50 \% \mathrm{RH}\right)$, and we found a PEO thickness of $200 \pm 5 \mathrm{~nm}$. 


\section{Results and discussion}

The surface topography of the PEO thin films was studied under a humidity-controlled atmosphere. The samples were scanned in tapping mode using a dual-pass protocol. In the first pass, we obtained the topography of the thin films, using a tapping mode imaging procedure with an amplitude of $\sim 14 \mathrm{~nm}(360 \mathrm{mV})$. In Figures 1a-c, we show the topography of the PEO at 15,35 , and $50 \% \mathrm{RH}$ respectively. For all cases, we observed a continuous and rather smooth surface with the topographical characteristics of a semicrystalline polymer. As reported previously, these images suggest that the surface nanostructures assembled as flat-on lamellas. ${ }^{23-25}$ We observed no changes of the surface morphology upon humidity changed. Only at $50 \% \mathrm{RH}$, we noticed a slightly blurrier topography image, which might be attributed to capillary condensation.
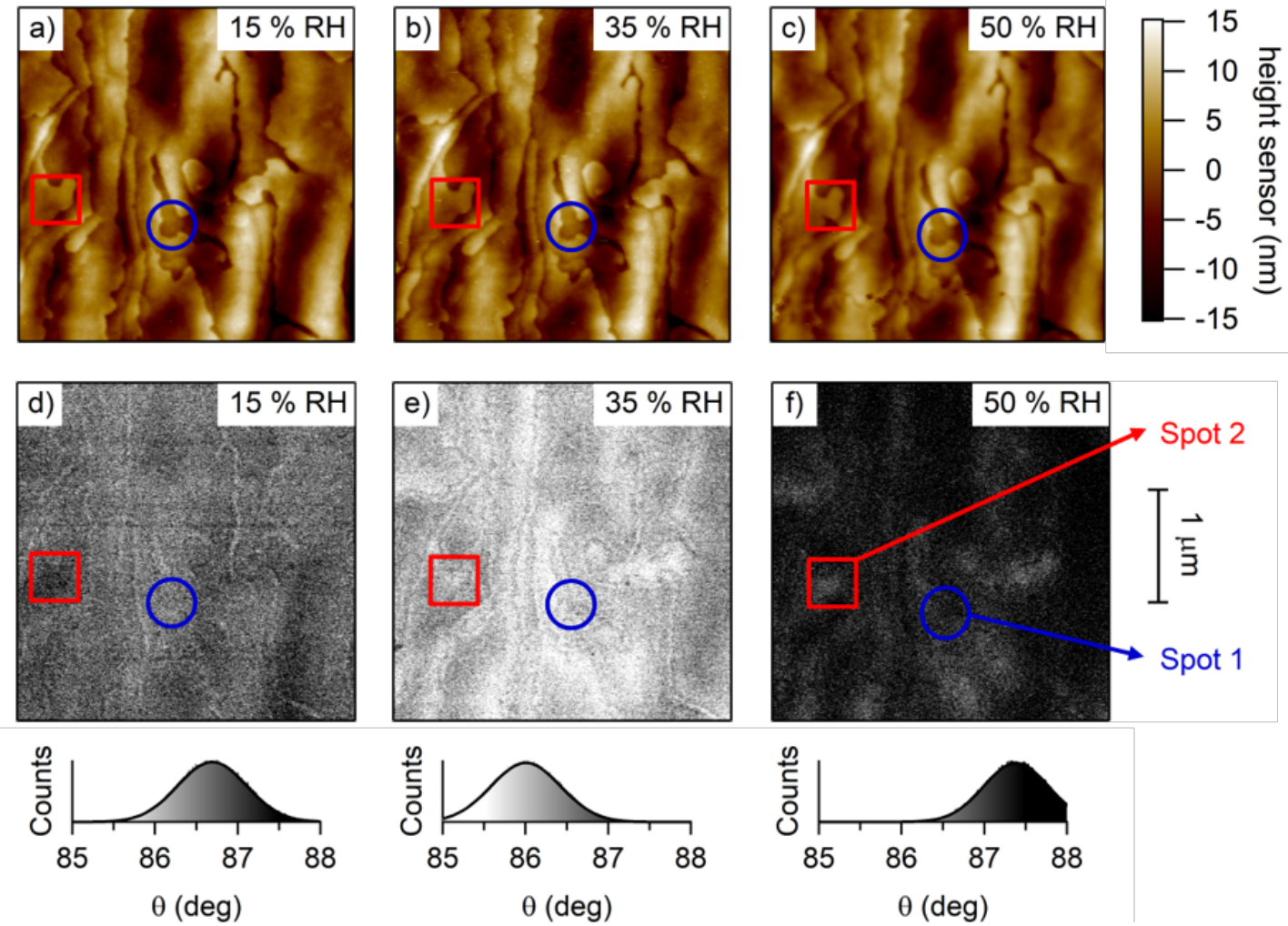

Figure 1: AFM topography of a PEO thin film at varying environmental humidity of a) $15, \mathrm{~b}) 35$, and c) $50 \% \mathrm{RH}$. Corresponding nanoDielectric images are shown below with 
image histograms. All color tables are scaled alike and tip positions for nanoDielectric spectroscopy are highlighted by spots 1 and 2 .

During the second pass, we recorded images with dielectric relaxation contrast, i.e., maps related to the second harmonic cantilever oscillation phase $(\theta)$. In this part of the imaging procedure, called nanoDielectric Imaging (nDI), ${ }^{26}$ we set a constant tip-sample distance of $16 \mathrm{~nm}$ and the surface topography was re-scanned with the mechanical cantilever excitation disabled and applying a sinusoidal bias to the probe $\left(6.8 \mathrm{~V}_{\mathrm{RMS}}\right.$, at a frequency $f=1 \mathrm{kHz}$ ). The resulting cantilever deflection signal was routed to the lock-in amplifier for analysis. A rather small tip-sample distance was chosen to enhance the phase shift contrast during imaging, regarding the data acquisition rate.

Figures $1 \mathrm{~d}$-f show the nDI phase maps obtained by this technique. Every map yielded a contrast at fixed humidity that has been transformed into a histogram of $\theta$-values (lower frame in Figure 1). We observed that the $\theta$-values fell within a 2-degree range, with an average clearly below 90 degrees, i.e., below that value corresponding to a situation without dielectric relaxations. By varying the relative humidity, the distribution of $\theta$ values shifted in a non-monotonous way, leading to a change in the maps' contrast. In detail, as we increased \% RH from 15 to $35 \%$, the distribution maximum shifted towards lower $\theta$ values. However, as we kept increasing towards $50 \% \mathrm{RH}$, the distribution shifted back to higher $\theta$ values, even higher than those recorded at $15 \% \mathrm{RH}$. The nDI maps demonstrated that the PEO thin films presented humidity-dependent dielectric properties.

For semicrystalline polymers, the dielectric properties of the film are determined to a large extent by the conductivity and permittivity of the crystalline as well as the amorphous phases (see Supporting Information, and $\operatorname{ref}^{11}$ ). The superposition of both 
contributions results in a frequency-dependent effective dielectric permittivity of the film, characterized by two principal quantities: the relaxation strength that measures the change in permittivity from the high to the low frequency limits, and the characteristic frequency around which the change occurs that defines the relaxation time. ${ }^{11}$ Therefore, the different values of $\theta$ within the nDI maps have to be related to changes of either of these two factors, or a combination of both. However, from the maps shown in Figures 1d-f no further conclusions can be drawn, as they are based on a single electric field frequency response. In order to evaluate the frequency dependent thin film permittivity, we performed nanoDielectric Spectroscopy (nDS) measurements.

In $\mathrm{nDS}$ experiments, we detected the phase shift of the cantilever motions, when sweeping the electrical excitation frequency $\left(6.8 \mathrm{~V}_{\mathrm{RMS}}, 1 \leq f(\mathrm{~Hz}) \leq 10^{5}\right)$ at fixed locations on the polymer film surface. Further details of the nDS procedure are given in the Supporting Information. The $\mathrm{nDS}$ experiments were performed at two distinct areas of the films for three different humidity conditions. We highlighted these spots with red squares and blue circles in Figure 1. The two regions corresponded to higher and lower $\theta$-values in the nDI maps. In Figure 2, we present the corresponding collected spectra. The lower frequency limit was selected at $5 \mathrm{~Hz}$. Below this frequency, the nDS spectra noise-to-signal ratio was high, precluding data reliability. These plots show the phase shift $(\Delta \theta)$ vs $f$, which was evaluated as $\Delta \theta=\theta_{\mathrm{REF}}-\theta$, where $\theta_{\mathrm{REF}}\left(\right.$ around $\left.90^{\circ}\right)$ depends on the mechanical characteristics of the AFM probe and of the electronics used for detecting the oscillations. This frequency dependence of $\theta_{\text {REF }}$ was determined by following the equivalent procedure on poly(vinyl acetate) polymer film (500 nm thick), which is free of dielectric losses at room temperature. We have also confirmed that the so obtained $\theta_{\text {REF }}$ is not significantly affected by humidity (Supporting Information). 

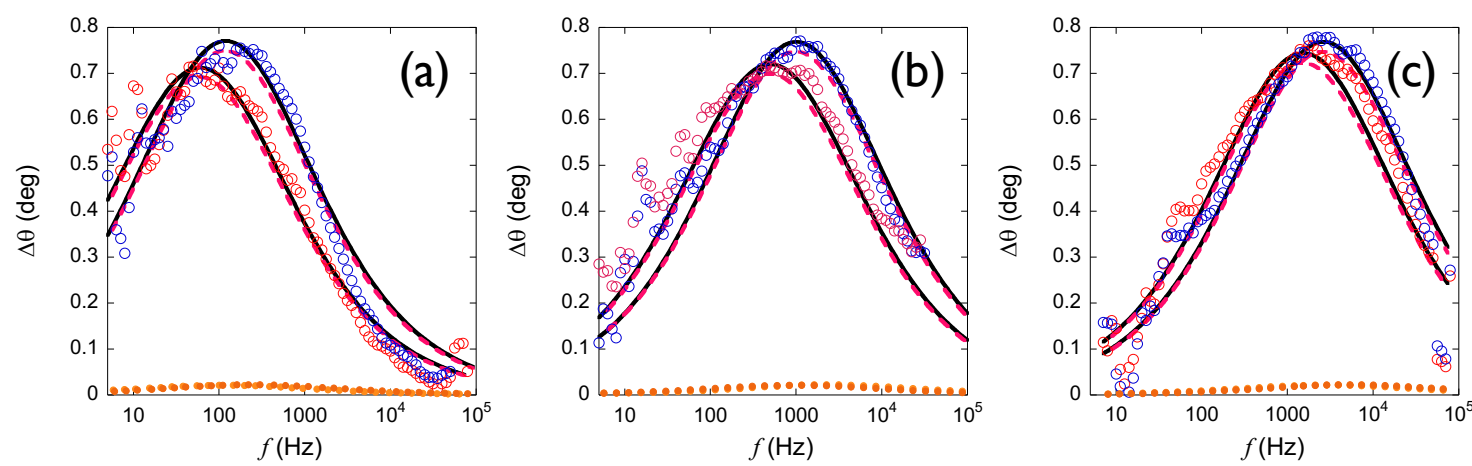

Figure 2: nDS spectra of PEO at two selected surface spots at a) $15 \% \mathrm{RH}$, b) $35 \% \mathrm{RH}$, and c) $50 \% \mathrm{RH}$ environmental humidity. The different lines correspond to data modelling as detailed in the manuscript, and show the contributions from the tip (dashed), the cantilever (dotted), and their sum (solid).

Figure 2 shows that $\% \mathrm{RH}$ variations greatly affected the measured $\mathrm{nDS}$ signal, in agreement with the $\mathrm{nDI}$ maps. In addition, for a fixed \% $\% \mathrm{RH}$, we observed that the position and intensity of the peaks were different for the two analyzed spots (red squares vs blue circles in Figure 2). At spot 1 (red squares), we measured a lower relaxation intensity and slower characteristic time, compared to spot 2 (blue circles). The differences were not pronounced but systematic. Also, the values of the phase shift in the nDS experiments were moderate. We attribute this last observation to the fact that the tipsample distance in $\mathrm{nDS}$ is relatively large, which results in a relatively high relevance of the cantilever contribution to the interaction force. The latter is nearly insensitive to the film dielectric properties. ${ }^{22}$ Measurements at such large distances were necessary to guarantee a proper tip-sample interaction of pure electrostatic nature and no relevant $Z$ piezo movement during the spectra recording. A strict control on these parameters is of utmost importance for generating high quality data. It should be pointed out that in our previous report on PEO thin films under dry conditions we were not able to resolve distinct $\mathrm{nDS}$ spectra at different probed locations. ${ }^{9}$ Considering that there are no other 
major differences, most probably, the improved experimental approach is the reason for this difference.

Following literature reports, ${ }^{9}$ the measured phase shift results from a frequencydependent capacitance:

$$
\Delta \theta(\omega)=\tan ^{-1}\left\{\operatorname{Im}\left|\frac{\partial C^{*}(\omega)}{\partial z}\right| / \operatorname{Re}\left|\frac{\partial C^{*}(\omega)}{\partial z}\right|\right\}
$$

In order to analyze the $\Delta \theta(\omega)$ measurements quantitatively, the probe-sample capacitance, $C^{*}(\omega)$, has been modeled by considering the contributions from the tip apex and the cantilever separately (Supporting Information).

At room temperature, the effective dielectric permittivity function $\left(\varepsilon^{*}(\omega)\right)$ of the semicrystalline PEO thin film mainly originates from ion-trapping processes at the interfaces. The resulting dielectric relaxation is referred to as Maxwell-Wagner-Sillars (MWS) relaxation, which is a well-known process in the literature. ${ }^{11,12}$ For the PEO film case, $\varepsilon^{*}(\omega)$ can be expressed in terms of the conductivity $(\sigma)$ and dielectric constant $(\varepsilon)$ of both the amorphous and crystalline phases, in respect to their respective volume fractions (Supporting Information). In this work, we followed a previously reported approach that assumed a flat-on layered structure with a non-conducting crystalline phase, separated by conductive amorphous layers. ${ }^{9},{ }^{11}$ In the following modelling, it was assumed that the amorphous and crystalline phases have frequencyindependent permittivities in the explored range, $\varepsilon_{a}=3.0, \varepsilon_{c}=2.5 .^{9}$ As previously reported, in order to describe the experimental data using this approach, a distribution of conductivities in the amorphous phase should be used. ${ }^{9}$ Such a distribution would reflect the very heterogeneous character of the segmental mobility in the amorphous 
phase of semicrystalline polymers. ${ }^{27}$ In particular, we could demonstrate that a Gaussian distribution of $\log _{10} \sigma$ described well the nDS data of the PEO thin films. ${ }^{9}$

The different lines in Figure 2 show the modeling of the experimental data, following the approach outlined above. As fitting parameters, we used the crystalline fraction $(\varphi)$, and the mean and variance of the log-conductivity distributions of the amorphous phase $\left(\log \sigma_{\mathrm{m}}\right.$ and $\log _{-} v a r \sigma_{\mathrm{m}}$ respectively). For a film thickness of $\sim 150 \mathrm{~nm}$, the dominant contribution to the nDS spectra arose from the tip (dashed lines in Figure 2), while the cantilever-related signal was, as expected, almost zero for all cases (dotted lines in Figure 2). This finding is important as it allowed us to use the local dielectric function for both the local and average film permittivities in the modeling process (Supporting Information).

The resulting fitting parameters are presented in Figure 3 as a function of $\% \mathrm{RH}$. We found that the values of the crystalline fraction were essentially independent of $\% \mathrm{RH}$, as expected (Figure 3a). The obtained $\varphi$ value is similar to the $38 \%$ crystallinity recently reported by Wang and collaborators, by macroscopic methods, for a spin-casted PEO thin film of similar thickness. ${ }^{28}$ Interestingly, we detected small but measurable differences in $\varphi$ when comparing the two probed spots for each $\% \mathrm{RH}$. This suggests that in the spots showing higher $\varphi$ values, the crystals would be better developed. The presence of different crystalline regions cannot be interpreted from the topography images, solely. Thus, the difference in the crystalline fraction is at least one of the factors originating the contrast in the $\mathrm{nDI}$ maps, where darker areas correspond to zones with higher crystalline fractions.

The other two parameters obtained from the nDS fittings, are related to the conductivity behavior in the amorphous phase. We found that the variance of the log-conductivity 
distributions was insensitive to $\% \mathrm{RH}$, as well as to the probed position on the film (log_var $\sigma_{\mathrm{m}}=0.8$ decades). By contrast, the mean log-conductivity $\left(\log \sigma_{\mathrm{m}}\right)$ was the only parameter clearly depending on $\% \mathrm{RH}$ (see Figure $3 b$ ). We obtained higher conductivity values in the amorphous phase for the positions where the crystalline fraction was lower. This result can be understood by taking into account that the amorphous PEO segments surrounding well-developed crystals would present more constrained dynamics. ${ }^{27}$
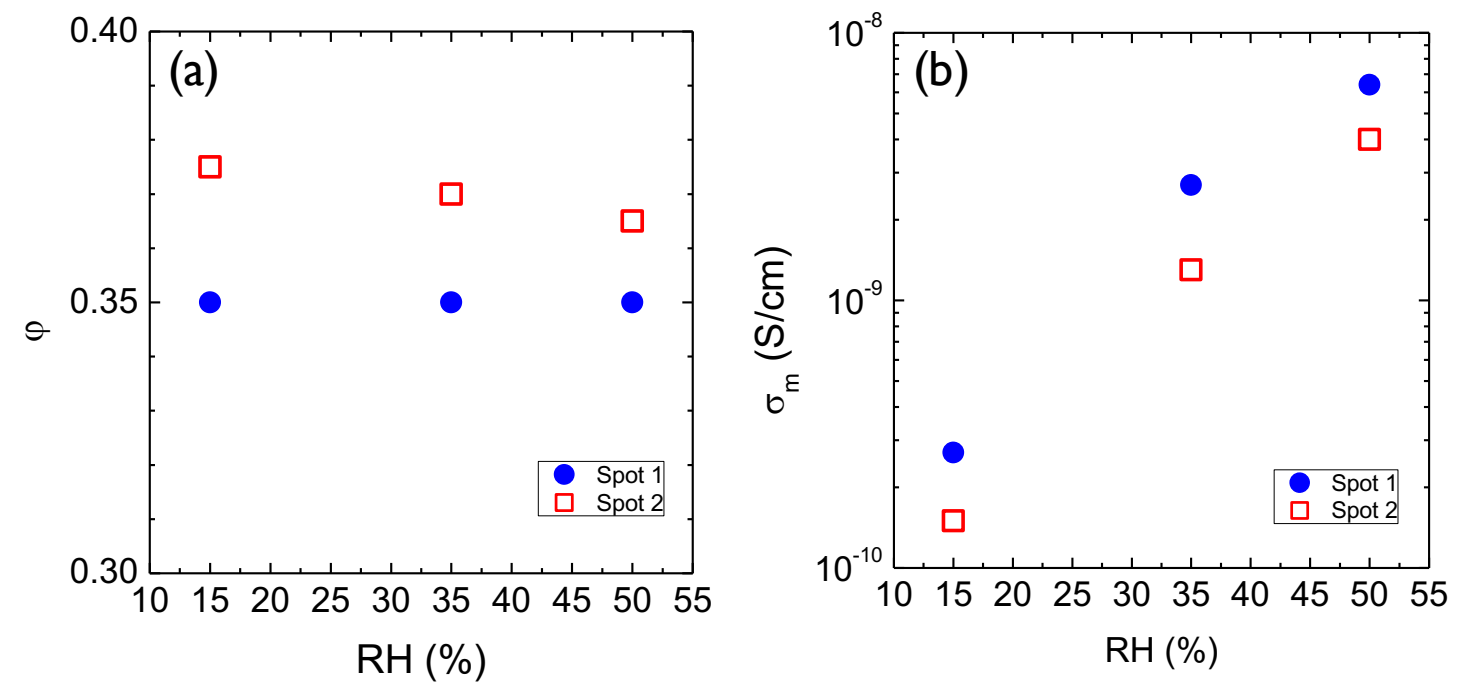

Figure 3: Fitting parameters obtained from $\mathrm{nDS}$ modeling, as a function of relative humidity. (a) Crystalline fraction $(\varphi)$. (b) Mean conductivity of the PEO amorphous phase $\left(\sigma_{\mathrm{m}}\right)$. Error bars are smaller than the symbol size.

It is important to point out that the overall PEO film conductivity is much smaller than the one deduced for the amorphous phase, and it is below the detection limit of the $\mathrm{nDS}$ experiments $\left(\sim 10^{-12} \mathrm{~S} / \mathrm{cm}\right)$. This low value results most-likely from the blocking effect of the PEO crystals on the ionic transport. ${ }^{7,8}$ Moreover, by extrapolating the obtained conductivity values in the amorphous phase of the semicrystalline thin films to zero humidity, we can deduce a reduced conductivity as compared with the conductivity value deduced by extrapolating the PEO-melt conductivity to room temperature $\left(\sim 10^{-7}\right.$ 
$\mathrm{S} / \mathrm{cm}) .{ }^{9}$ This result is based on the fact that the amorphous phase in a semicrystalline polymer has very distinct characteristics compared to those of the pure amorphous polymer. ${ }^{27}$ In particular, a much hindered segmental dynamics exists in semicrystalline polymers, and it is well established that mobility is directly coupled with the ionic transport. $^{29}$

Here, the major influence of humidity has been a significant effect on the characteristic frequency of the nDS signal. In turn, this results in a corresponding dramatic change in the conductivity of the amorphous phase of the semicrystalline PEO thin films. We found that just by changing the atmosphere humidity from 15 to $50 \% \mathrm{RH}, \sigma_{\mathrm{m}}$ increased by a factor close to 25 . In fact, it is well documented that humidity yields a substantial increase of the conductivity of electrolytes based on amorphous PEO ${ }^{29,}{ }^{30}$ For instance, Kovacs et al. reported an increase of the DC-conductivity of about 1.5 decades in fully amorphous plasticized PEO electrolytes, when comparing experiments performed in an Ar atmosphere with those for samples exposed to moisture. ${ }^{29}$ Similar results were reported by Maranas et al. when comparing the conductivity of different PEO based nanoparticle-filled electrolytes obtained at low and high humidity conditions. ${ }^{30}$ It has been argued that there are two major reasons for these observations. On the one hand, the humidity acts as a plasticizer facilitating the motions of the PEO segments and consequently improving ion transport. ${ }^{29}$ On the other hand, the interaction between ether groups of PEO and the metal cations, arising from impurities or added salts, is lowered by the presence of water, due to its high dielectric constant ${ }^{31}$. Thus, an increasing number of cations becomes available for ionic transport.

While here un-doped PEO thin films have been studied, the reasons for the strong effect of atmosphere humidity on the conductivity of the amorphous phase are probably similar to those discussed above. The conductivity in un-doped PEO is also dominated 
by the presence of ionic impurities, which are uncontrolled and originate from the synthesis and processing of the polymer. The fact that the humidity modifies the conductivity of PEO electrolytes with different amount of salt in a similar manner would explain why we found also 1.4 decades difference between the values of conductivity in the amorphous phase from the lowest to the highest humidity conditions. $^{29}$

\section{Conclusions}

We studied the dielectric properties of PEO thin films under controlled relative humidity conditions $(15-50 \% \mathrm{RH}$ range) by scanning probe techniques. We found no changes in the PEO film topography with humidity. However, we observed a humidity dependent dielectric contrast for different regions of the PEO thin film and a strong influence of the humidity conditions on the dielectric properties of PEO thin films. Based on the dielectric contrast maps, we performed local dielectric measurements. This technique allowed probing the dielectric properties of the PEO thin films with lateral resolution in the nanometer range. Modeling of these $\mathrm{nDS}$ results showed that the crystallinity of the PEO thin films and the conductivity of their amorphous phase play center roles. The crystalline fraction of the polymer was found to be about $36 \%$, with almost no variations between the probed areas and without humidity dependence. The conductivity of the amorphous phase showed measurable differences and a strong humidity dependence. The conductivity variation was understood as a result of an increasing number of cations due to the presence of surrounding water molecules. Our findings on un-doped PEO are also of high relevance for doped systems, like those found in batteries. Relative humidity leads to a high solubility of impurities and a high mobility of the ions. Moreover, it is not only the total concentration of crystalline areas but also their distribution which has major influence on the overall conductivity. 


\section{Conflicts of interest}

There are no conflicts to declare.

\section{Acknowledgements}

This work was supported by the European Union: EUSMI, H2020-INFRAIA-2016-1,

PROJECT 731019. A.A. acknowledges funding from Spanish Government "Ministerio de Ciencia, Innovacion y Universidades" (PGC2018-094548-B-I00 (MCIU/AEI/FEDER, UE)), and Basque Government (IT-1175-19). D.E.M. acknowledges financial support via the "Juan de la Cierva - Incorporación" grant (IJCI2017-31600, MCIU - Spain).

\section{References}

1. F. Croce, G. B. Appetecchi, L. Persi and B. Scrosati, Nature, 1998, 394, 456-458.

2. M. Armand, Advanced Materials, 1990, 2, 278-286.

3. J. M. Tarascon and M. Armand, Nature, 2001, 414, 359-367.

4. M. M. Elmahdy, K. Chrissopoulou, A. Afratis, G. Floudas and S. H. Anastasiadis, Macromolecules, 2006, 39, 5170-5173.

5. P. Dhatarwal, R. J. Sengwa and S. Choudhary, Composites Communications, 2017, 5, 17.

6. R. J. Sengwa and S. Choudhary, Journal of Applied Polymer Science, 2014, 131.

7. Y. Jiang, X. Yan, Z. Ma, P. Mei, W. Xiao, Q. You and Y. Zhang, Polymers, 2018, 10, 1237.

8. Z. Xue, D. He and X. Xie, Journal of Materials Chemistry A, 2015, 3, 19218-19253.

9. D. E. Martínez-Tong, L. A. Miccio and A. Alegria, Soft Matter, 2017, 13, 5597-5603.

10. D. Zhou, D. Shanmukaraj, A. Tkacheva, M. Armand and G. Wang, Chem, 2019, 5, 23262352.

11. F. Kremer and A. Schönhals, Broadband Dielectric Spectroscopy, Springer, 2003.

12. M. Samet, V. Levchenko, G. Boiteux, G. Seytre, A. Kallel and A. Serghei, The Journal of Chemical Physics, 2015, 142, 194703.

13. H. W. Kammer, lonics, 2018, 24, 1415-1428.

14. P. S. Crider, M. R. Majewski, J. Zhang, H. Oukris and N. E. Israeloff, Applied Physics Letters, 2007, 91, 013102.

15. C. Riedel, R. Sweeney, N. E. Israeloff, R. Arinero, G. A. Schwartz, A. Alegria, P. Tordjeman and J. Colmenero, Applied Physics Letters, 2010, 96, 213110.

16. G. A. Schwartz, C. Riedel, R. Arinero, P. Tordjeman, A. Alegría and J. Colmenero, Ultramicroscopy, 2011, 111, 1366-1369.

17. R. Berger, H.-J. Butt, M. B. Retschke and S. A. L. Weber, Macromolecular Rapid Communications, 2009, 30, 1167-1178.

18. C. Riedel, A. Alegría, G. A. Schwartz, R. Arinero, J. Colmenero and J. J. Sáenz, Applied Physics Letters, 2011, 99, 023101. 
19. L. C. Fernandes, D. M. Correia, N. Pereira, C. R. Tubio and S. Lanceros-Méndez, ACS Applied Polymer Materials, 2019, DOI: 10.1021/acsapm.9b00675.

20. D. T. W. Toolan, A. Isakova, R. Hodgkinson, N. Reeves-McLaren, O. S. Hammond, K. J. Edler, W. H. Briscoe, T. Arnold, T. Gough, P. D. Topham and J. R. Howse, Macromolecules, 2016, 49, 4579-4586.

21. L. A. Miccio, M. M. Kummali, G. A. Schwartz, Á. Alegría and J. Colmenero, Ultramicroscopy, 2014, 146, 55-61.

22. L. A. Miccio, M. M. Kummali, G. A. Schwartz, Á. Alegría and J. Colmenero, Journal of Applied Physics, 2014, 115, 184305.

23. J. M. Schultz and M. J. Miles, Journal of Polymer Science Part B: Polymer Physics, 1998, 36, 2311-2325.

24. B. Crist and J. M. Schultz, in Encyclopedia of Polymers and Composites, ed. S. Palsule, Springer Berlin Heidelberg, Berlin, Heidelberg, 2021, DOI: 10.1007/978-3-642-371790_23-1, pp. 1-25.

25. B. Crist and J. M. Schultz, Progress in Polymer Science, 2016, 56, 1-63.

26. C. Riedel, R. Arinero, P. Tordjeman, G. Lévêque, G. A. Schwartz, A. Alegria and J. Colmenero, Physical Review E, 2010, 81, 010801.

27. A. Alegria and J. Colmenero, Soft Matter, 2016, 12, 7709-7725.

28. Y. Wang, K. Gu, X. Monnier, H. Jeong, M. Chowdhury, D. Cangialosi, Y.-L. Loo and R. D. Priestley, ACS Macro Letters, 2019, 8, 1115-1121.

29. M. Kovač, M. Gaberšček and S. Pejovnik, Solid State lonics, 2000, 131, 323-327.

30. S. K. Fullerton-Shirey, L. V. N. R. Ganapatibhotla, W. Shi and J. K. Maranas, Journal of Polymer Science Part B: Polymer Physics, 2011, 49, 1496-1505.

31. A. Johansson, A. Lauenstein and J. Tegenfeldt, The Journal of Physical Chemistry, 1995, 99, 6163-6166. 